\title{
IMPROVEMENT OF TAKAGI-SUGENO FUZZY MODEL FOR THE ESTIMATION OF NONLINEAR FUNCTIONS
}

\author{
Agustín Jiménez, Basil M. Al-Hadithi, Fernando Matia, and Rodolfo Haber-Haber
}

\begin{abstract}
Two new and efficient approaches are presented to improve the local and global estimation of the Takagi-Sugeno (T-S) fuzzy model. The main aim is to obtain high function approximation aceuracy and fast convergence. The main problem is that the T-S identification method can not be applied when the membership functions are overlapped by pairs. The approaches developed here can be considered as generalized versions of T-S method with optimized performance. The first uses the minimum norm approach to search for an exact optimum solution at the expense of increasing complexity and computational cost. The second is a simple and less computational method, based on weighting of parameters. Illustrative examples are chosen to evaluate the potential, simplicity and remarkable performance of the proposed methods and the high accuracy obtained in comparison with the original T-S model.
\end{abstract}

Key Words: Nonlinear systems, fuzzy systens, Takagi-Sugeno fuzzy model, universal approximators, optimization.

\section{INTRODUCTION}

Nonlinear control systems based on the TakagiSugeno (T-S) fuzzy model [1] have attracted lots of attention during the last twenty years (e.g., see [2-11]). They provide a powerful solution for development of function approximation, systematic techniques to stability analysis and controller design of fuzzy control systems in view of fruitful conventional control theory and techniques. They also allow relatively easy application of powerful learning techniques for their identification from data. This model is formed by using a set of fuzzy rules to represent a nonlinear system as a set of local affine models which are connected by fuzzy membership functions. This fuzzy modelling method presents an alternative technique to represent complex nonlinear systems and [12] and reduces the number of rules in modelling higher order nonlinear systems [1] and [6].

Some papers focus on the proof of the universal approximation property of T-S fuzzy models as they are able to approximate any smooth nonlinear functions to any degree of accuracy in any convex compact region [12-14]. It was clearly shown, however, that the number of fuzzy rules increases as the approximation error tends to zero [15]. This exponential growth cannot be eliminated, so it becomes difficult to make use of the universal approximation property of TS fuzzy modelling for practical purposes. In addition to that, if the number of rules is bounded, the resulting set of functions is nowhere dense in the space of approximated functions [16]. These conflicting objectives have motivated researchers to find a balance between the specified accuracy and the computational complexity of the resulting fuzzy model.

Recently, great interest has been shown in tensor product distributed compensation (TPDC) as an 
established controller design framework, that links TP model transformation and parallel distributed compensation (PDC) framework. TP model transformation converts different models to a common representational form [17]. In [18] the approximation capabilities of TP model forms is analyzed, because the universal applicability of TPDC framework strongly relies on it. Consequently, in a class of control problems this property requires the usage of tradeoff techniques between the accuracy and the complexity of the TP form, which is easily feasible within the TPDC framework unlike in analytic models.

In [19] the higher order singular-valuedecomposition (HOSVD) based canonical form of linear parameter varying models is presented and a modeless most important invariant characteristics is developed. The numerical reconstructibility of the canonical form using a routinely executable tractable uniform method is analyzed. Convergency theorems for given numerical reconstruction constraint are presented.

In [20] a singular value-based method for reducing a given fuzzy rule set is developed. The method conducts singular value decomposition of the rule consequents and generates certain linear combinations of the original membership functions to form new ones for the reduced set. The present work characterizes membership functions by the conditions of sum normalization ( $\mathrm{SN}$ ), nonnegativeness (NN), and normality (NO). Algorithms to preserve the $\mathrm{SN}$ and NN conditions in the new membership functions are presented. The present work discusses three specific applications of fuzzy reduction: fuzzy rule base with singleton support, fuzzy rule base with nonsingleton support and the T-S model.

In [21], the authors proposed to obtain the best features of Mamdani and Takagi-Sugeno models by using an affine global model with function approximation capabilities which maintains local interpretation. The suggested model is composed of variant coefficients which are independently governed by a zeroth order fuzzy inference system. This model may be interpreted as a generalization of the T-S model in which dynamics coefficients have been decoupled. They have shown that Mamdani and Takagi-Sugeno models can be combined so that local and global interpretations are preserved.

Great attention has been paid to the identification of T-S fuzzy models and several results have been obtained $[22,13,23]$. They are based upon two kinds of approaches, one is to linearize the original nonlinear system in various operating points when the model of the system is known, and the other is based on the input-ontput data collected from the original nonlinear system when its model is unknown.
The authors in [22] use a fuzzy clustering method to identify T-S fuzzy models, including identification of the number of fuzzy rules and parameters of fuzzy membership functions, and identification of parameters of local linear models by using a least squares method $[24,25]$. The goal is to minimize the error between T-S fuzzy models and the corresponding original nonlinear systems. In [26]. Klawonn et al. explained how fuzzy clustering techniques could be applied to design a fuzzy controller from the training data. In [27]. Hong and Lee have analyzed that the disadvantages of most fuzzy systems are that the membership functions and fuzzy rules should be predefined to map numerical data into linguistic terms and to make fuzzy reasoning work. They suggested a method based on the fuzzy clustering technique and the decision tables to derive membership functions and fuzzy rules from numerical data. However. Hong and Lee's algorithm presented in [27] needs to predefine the membership functions of the input linguistic variables and it simplifies fuzzy rules by a series of merge operations. As the number of variables becomes larger, the decision table will grow tremendously and the process of the rule simplification based on the decision tables becomes more complicated. The authors in [13] suggest a method to identify T-S fuzzy models. Their method aims at improving the local and global approximation of the T-S model. However, this complicates the approximation in order to obtain both targets. It has been shown that constrained and regularized identification methods may improve interpretability of constituent local models as local linearizations, and locally weighted least squares method may explicitly address the trade-off between the local and global accuracy of T-S fuzzy models.

In [24] a method of interval fuzzy model identification was developed. The method combines a fuzzy identification methodology with some ideas from linear programming theory. The idea is then extended to modelling the optimal lower and upper bound functions that define the band which contains all the measurement values. This results in lower and upper fuzzy models or a fuzzy model with a set of lower and upper parameters. This approach can also be used to compress information in the case of a large amount of data and in the case of robust system identification. The method can be efficiently used in the case of the approximation of the nonlinear functions family. The paper focuses on the development of an interval L-norm function approximation methodology problem using the LP technique and the TS fuzzy logic approach. This results in lower and upper fuzzy models or a fuzzy model with lower and upper parameters. 
In [3] a constructive method to synthesize a multiple input single output (MISO) TS fuzzy logic system imposing the requested derivative constraints on the function representing its behaviour is presented. The values of that function and its partial derivatives on the grid points of the input space permit definition of a suitable interpolator of the function itself.

In [28], a new approach to fuzzy modelling using the relevance vector learning mechanism (RVM) based on a kernel-based Bayesian estimation is introduced. The main concern is to find the best structure of the TS fuzzy model for modelling nonlinear dynamic systems with measurement error. The number of rules and the parameter values of membership functions can be found as optimizing the marginal likelihood of the RVM in the proposed FIS. Because the RVM is not necessary to satisfy Mercer's condition, selection of kernel function is beyond the limit of the positive definite continuous symmetric function of SVM. The relaxed condition of kernel function can satisfy various types of membership functions in fuzzy model. The RVM which was compared with support vector learning mechanism in examples had the small model capacity and described good generalization.

In [29] an approach for fuzzy approximation of continuous function on a compact domain is developed. The approach calls for sampling the function over a set of rectangular grid points and applying singular value decomposition to the sample matrix. The resulting quantities are then adapted to become rule consequents and membership functions via the conditions of sum normalization (SN) and non-negativeness (NN). The membership functions and corresponding rule consequents are not needed to be predefined, rather they are extracted directly from the function samples. Tradeoff between the number of membership functions and the desired approximation accuracy is also discussed.

In [30] the authors suggest a transformation method that offers a trade-off between the modelling complexity and accuracy of the T-S fuzzy model. The suggested transformation method is aimed at finding the minimal number of fuzzy rules for a given accuracy of a given T-S fuzzy model. Optimization of the approximation trade-off is conceptually obtained by the discarding of those rules, which have weak or no contribution at all to the output.

Interpolation is an important subject for reducing dense rule bases as well for deriving useful information from sparse ones. In [15] the authors show that rule interpolation helps in reducing the identification complexity as it allows rule bases with gaps. It is suggested that only the minimal necessary number of rules remain which contain the essential information, and all other rules are replaced by the interpolation algorithm.

The approach proposed in [15] has some drawbacks such as subnormal conclusion for certain configuration of the involved fuzzy sets and does not always lead to interpretable fuzzy membership functions. Nevertheless, it is characterized by having the lowest computational complexity and has good approxiamtion aspects comparing with other fuzzy rule interpolation techniques. In [31], a new technique for fuzzy rule interpolation was investigated. The aim is to combine the advantageous computational behavior of $[15]$ and at the same time reduce the disadvantage of subnormality.

In spite of its advantageous properties in various approximation aspects and in complexity reduction, it was shown that $\alpha$-cut distance based fuzzy rule base interpolation has some essential deficiencies, for instance, it does not always result in immediately interpretable fuzzy membership functions. In [32], an approach is presented to get rid of these disadvantages. It is based on the interpolation of relations instead of interpolating $\alpha$-cut distances, and which offers a way to derive a family of interpolation methods capable of eliminating some typical deficiencies of fuzzy rule interpolation techniques.

In [33] a generalized version of a previous Cartesian approach [34] and [35] for interpolating fuzzy rules of membership functions with finite number of characteristic points is presented. Instead of representing membership functions as points in Cartesian spaces, they now become elements in the space of square, integrable function. Interpolation is thus made between the antecedent and consequent function spaces. The generalized representation allows an extended class of membership functions satisfying two monotonicity conditions to be accommodated in the interpolation process.

In [1], the atthors develop an interesting method to identify nonlinear systems using input-output data. They divide the identification process into three steps; premise variables, membership functions and consequent parameters. With respect to membership functions, they apply a nonlinear programming technique using the complex method for the minimization of the performance index.

In spite of such important works, prior to 1989 , most FLSs were still designed with preselected structures and the adjustment of membership functions was carried out by trial and enror. Since 1989 , a number of techniques for structure and/or parameter identification from I/O data have been suggested in the literature. In 1989. Sugeno and Tanaka presented a method for a successive identification method for constructing a 
T-S fuzzy model [24]. In this method, structure determination was implemented by a combination of leastsquares estimation (LSE), the complex method, and an unbiasedness criterion; while parameter adjustment was achieved using fuzzy adjustment rules and a weighted recursive least-squares algorithm.

In 1991. Wang and Mendel developed a method for generating fuzzy rules by learning from examples [25] and proved that a fuzzy inference system is a universal approximator by the Stone-Weierstrass theorem [36].

In 1995, Wang proposed a new state-space analytical approach to fuzzy identification of nonlinear dynamical systems [37]. In 1996, Langari and Wang proposed achieving structure identification of a T-S fuzzy model by using a combination of a fuzzy cmeans clustering technique and a fuzzy discretization technique [38].

In [39], Nozaki et al. presented a heuristic method for generating T-S fuzzy rules from numerical data, and then converted the consequent parts of T-S fuzzy rules into linguistic representation.

In [40], a study has outlined a new min-max approach to the fuzzy clustering, estimation, and identification with uncertain data. The proposed approach minimizes the worst-case effect of data uncertainties and modelling enrors on estimation performance without making any statistical assumption and requiring a priori knowledge of uncertainties.

A new fuzzy system containing a dynamic rule base is proposed in [41]. The characteristic of the proposed system is in the dynamic nature of its rule base which has a fixed number of rules and allows the fuzzy sets to dynamically change or move with the inputs. The number of the rules can be small, and chosen by the designer. The proposed system is capable of approximating any continuous function on an arbitrarily large compact domain and any uniformly continuous function on infinite domains. This paper addresses existence conditions, and as well providing constructive sufficient conditions so that the new fuzzy in the field of stability and controller synthesis are based on this type of membership function. Moreover, the methods presented here are characterized by the high accuracy obtained in approximating nonlinear systems locally and globally in comparison with the original T-S model. The rest of the paper is organized as follows. In Section II, the estimation of Fuzzy T-S Model's parameters is described. Section III presents the restrictions of T-S identification method. Section IV demonstrates the proposed approaches to improve and generalize the T-S model. Section V entails various examples to demonstrate the validity of the proposed approaches. These examples show that the proposed approaches are less conservative than those based on the (standard) T-S model and illustrate the utility of the proposed approaches in comparison with the T-S model. The conclusions of the effectiveness and validity of the proposed apprached are explained in Section VI.

\section{ESTIMATION OF FUZZY T-S MODEL'S PARAMETERS}

An interesting method of identification is presented in [1]. The idea is based on estimating the nonlinear system parameters minimizing a quadratic performance index. The method is based on the identification of functions of the following form:

$$
\begin{aligned}
& f: \Re^{n} \longrightarrow \Re \\
& y=f\left(x_{1}, x_{2}, \ldots, x_{n}\right) .
\end{aligned}
$$

Each IF-THEN rule $R^{i_{1} \ldots i_{n}}$, for an $n^{\text {th }}$ order system can be rewritten as follows:

$$
\begin{aligned}
& S^{\left(i_{1} \ldots i_{n}\right)}: \text { If } x_{1} \text { is } M_{1}^{i_{1}} \text { and } \ldots x_{n} \text { is } M_{n}^{i_{n}} \text { then } \\
& \hat{y}=p_{0}^{\left(i_{1} \ldots i_{n}\right)}+p_{1}^{\left(i_{1} \ldots i_{n}\right)} x_{1}+p_{2}^{\left(i_{1} \ldots i_{n}\right)} x_{2}+p_{n}^{\left(i_{1} \ldots i_{n}\right)} x_{n}
\end{aligned}
$$

where the fuzzy estimation of the output is:

$$
\hat{y}=\frac{\sum_{i_{1}=1}^{r_{1}} \ldots \sum_{i_{n}=1}^{r_{n}} w^{\left(i_{1} \ldots i_{n}\right)}(x)\left[p_{0}^{\left(i_{1} \ldots i_{n}\right)}+p_{1}^{\left(i_{1} \ldots i_{n}\right)} x_{1}\right]}{\sum_{i_{1}=1}^{r_{1}} \ldots \sum_{i_{n}=1}^{r_{n}} w^{\left(i_{1} \ldots i_{n}\right)}(x)}+\frac{\sum_{i_{1}=1}^{r_{1}} \ldots \sum_{i_{n}=1}^{r_{n}} w^{\left(i_{1} \ldots i_{n}\right)}(x)\left[p_{2}^{\left(i_{1} \ldots i_{n}\right)} x_{2}+p_{n}^{\left(i_{1} \ldots i_{n}\right)} x_{n}\right]}{\sum_{i_{1}=1}^{r_{1}} \ldots \sum_{i_{n}=1}^{r_{n}} w^{\left(i_{1} \ldots i_{n}\right)}(x)} .
$$

system can approximate any continuous function with bounded partial derivatives.

As we will demonstrate in this article, the T-S model can not be applied when the membership functions are overlapped by pairs. This limits the usage of the model because as it was shown in the last two decades that the major part of the results obtained
Let $\mathbf{m}$ be a set of input/output system samples $\left\{x_{1 k}, x_{2 k} \ldots, x_{n k}, y_{k}\right\}$. The parameters of the fuzzy system can be calculated as a result of minimizing a quadratic performance index:

$$
J=\sum_{k=1}^{m}\left(y_{k}-\hat{y}_{k}\right)^{2}=\|Y-X P\|^{2}
$$


where

$$
\begin{aligned}
Y & =\left[\begin{array}{llll}
y_{1} & y_{2} & \ldots y_{m}
\end{array}\right]^{T} \\
P & =\left[\begin{array}{lll}
P_{0}^{(1 \ldots 1)} & P_{1}^{(1 \ldots 1)} & P_{2}^{(1 \ldots 1)} \ldots P_{n}^{(1 \ldots 1)} \\
P_{0}^{\left(r_{1} \ldots r_{n}\right)} \ldots P_{n}^{\left(r_{1} \ldots r_{n}\right)} &
\end{array}\right] \\
X & =\left[\begin{array}{llll}
\beta_{1}^{(1 \ldots 1)} & \beta_{1}^{(1 \ldots 1)} x_{11} \ldots \beta_{1}^{(1 \ldots 1)} x_{n 1} \ldots \\
\beta_{1}^{\left(r_{1} \ldots r_{n}\right)} \ldots \beta_{1}^{\left(r_{1} \ldots r_{n}\right)} x_{n 1} & \\
\beta_{m}^{1 \ldots 1} & \beta_{m}^{(1 \ldots 1)} x_{1 m} \ldots \beta_{m}^{(1 \ldots 1)} x_{n m} \ldots \\
\beta_{m}^{\left(r_{1} \ldots r_{n}\right)} \ldots \beta_{m}^{\left(r_{1} \ldots r_{n}\right)} x_{n m}
\end{array}\right.
\end{aligned}
$$

and

$$
\beta_{k}^{\left(i_{1} \ldots i_{n}\right)}=\frac{w^{\left(i_{1} \ldots i_{n}\right)}\left(x_{k}\right)}{\sum_{i_{1}=1}^{r_{1}} \ldots \sum_{i_{n}=1}^{r_{n}} w^{\left(i_{1} \ldots i_{n}\right)}\left(x_{k}\right)} .
$$

If $\mathrm{X}$ is a matrix of complete rank, the solution is obtained as follows:

$$
\begin{aligned}
J & =\|Y-X P\|^{2}=(Y-X P)^{T}(Y-X P) \\
\nabla J & =X^{T}(Y-X P)=X^{T} Y-X^{T} X P=0 \\
P & =\left(X^{T} X\right)^{-1} X^{T} Y .
\end{aligned}
$$

\section{RESTRICTIONS OF T-S IDENTIFICATION METHOD}

The method proposed in [1] causes serious problems as it can not be applied in the most common case where the membership functions are those shown in Fig. 1. This means that, in the interval $\left[x_{i 1}, x_{i 2}\right]$, the membership functions are:

$$
\mu_{i 1}\left(x_{i}\right)=\frac{x_{i 2}-x_{i}}{x_{i 2}-x_{i 1}}, \quad \mu_{i 2}\left(x_{i}\right)=\frac{x_{i}-x_{i 1}}{x_{i 2}-x_{i 1}}
$$

which should verify:

$$
\begin{aligned}
\mu_{i 1}\left(x_{i 1}\right)=1 & \mu_{i 1}\left(x_{i 2}\right)=0 \\
\mu_{i 2}\left(x_{i 1}\right)=0 & \mu_{i 2}\left(x_{i 2}\right)=1 \\
\mu_{i 1}\left(x_{i}\right)+\mu_{i 2}\left(x_{i}\right)=1 . &
\end{aligned}
$$

For this case, which is widely used, it can easily be demonstrated [42] that the matrix $X$ is not of full rank

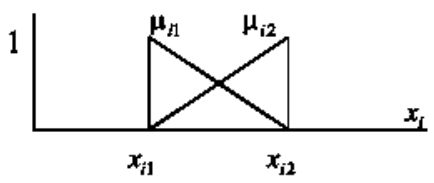

Fig. 1. Membership functions of the fuzzy system. and therefore $X^{T} X$ is not invertible, which makes the mentioned method of T-S invalid.

This result can be easily proven as follows:

Supposing that it exists:

$$
\begin{aligned}
& f: \Re^{n} \longrightarrow \Re \\
& y=f(x)
\end{aligned}
$$

in which each row of the matrix $\mathrm{X}$ is of the form:

$$
\begin{aligned}
X_{k} & =\left[\begin{array}{llll}
\mu_{k}^{1} & \mu_{k}^{1} x_{k} & \mu_{k}^{2} & \mu_{k}^{2} x_{k}
\end{array}\right] \\
& =\left[\begin{array}{lll}
\frac{x_{2}-x_{k}}{x_{2}-x_{1}} \frac{x_{2}-x_{k}}{x_{2}-x_{1}} x_{k} & \frac{x_{k}-x_{1}}{x_{2}-x_{1}} \frac{x_{k}-x_{1}}{x_{2}-x_{1}} x_{k}
\end{array}\right] .
\end{aligned}
$$

verifying that:

$$
\left[\frac{x_{2}-x_{k}}{x_{2}-x_{1}} \frac{x_{2}-x_{k}}{x_{2}-x_{1}} x_{k} \frac{x_{k}-x_{1}}{x_{2}-x_{1}} \frac{x_{k}-x_{1}}{x_{2}-x_{1}} x_{k}\right]\left[\begin{array}{l}
-x_{1} \\
1 \\
-x_{2} \\
1
\end{array}\right]=0 .
$$

The rank of $X$ in this case is 3 . In other words, the columns of $X$ are linearly dependent which in turn makes impossible the use of the above mentioned identification method proposed in [1]. Analyzing another example of two variables:

$$
\begin{aligned}
& f: \mathfrak{A}^{2} \longrightarrow \mathfrak{A}^{2} \\
& y=f\left(x_{1}, x_{2}\right) .
\end{aligned}
$$

Each row of the matrix $\mathrm{X}$ is of the form:

$$
\begin{aligned}
X_{k}= & {\left[\mu_{k}^{11} \mu_{k}^{21} \mu_{k}^{11} \mu_{k}^{21} x_{1 k} \mu_{k}^{11} \mu_{k}^{21} x_{2 k}\right.} \\
& \mu_{k}^{11} \mu_{k}^{22} \mu_{k}^{11} \mu_{k}^{22} x_{1 k} \mu_{k}^{11} \mu_{k}^{22} x_{2 k} \\
& \mu_{k}^{12} \mu_{k}^{21} \mu_{k}^{12} \mu_{k}^{21} x_{1 k} \mu_{k}^{12} \mu_{k}^{21} x_{2 k} \\
& \left.\mu_{k}^{11} \mu_{k}^{22} \mu_{k}^{12} \mu_{k}^{22} x_{1 k} \mu_{k}^{12} \mu_{k}^{22} x_{2 k}\right] .
\end{aligned}
$$

It can be noticed that the columns 1,3,4 and 6 have the same form as in the previous example multiplied by a constant $\mu_{k}^{11}$ and therefore they are linearly dependent as well. The same thing happens with the columns $6,9,10$ and 12, etc. In fact, the rank of the matrix in this case is 8 . The solution proposed in [1] avoids the occurrence of this situation. In order to identify a function in the interval $\left[x_{i 1}, x_{i 2}\right]$ using T-S method, certain intermediate points are chosen of the form:

$$
x_{i 1}^{*} \in\left[x_{i 1}, x_{i 2}\right] \text { and } x_{i 2}^{*} \in\left[x_{i 1}, x_{i 2}\right]
$$


and they use membership functions which verify:

$$
\begin{aligned}
& \mu_{i}^{\mathrm{l}}(x)= \begin{cases}\frac{x_{i}-x_{i 2}^{*}}{x_{i 1}-x_{i 2}^{*}} & x_{i 1} \leq x \leq x_{i 2}^{*} \\
0 & x_{i 2}^{*} \leq x \leq x_{i 2}\end{cases} \\
& \mu_{i}^{2}(x)= \begin{cases}0 & x_{i 1} \leq x \leq x_{i 1}^{*} \\
\frac{x_{i}-x_{i 1}^{*}}{x_{i 2}-x_{i 1}^{*}} & x_{i 1}^{*} \leq x \leq x_{i 2}\end{cases}
\end{aligned}
$$

and thus:

$$
\mu_{i}^{1}\left(x_{i 1}\right)=1, \mu_{i}^{1}\left(x_{i 2}^{*}\right)=0, \mu_{i}^{2}\left(x_{i 1}^{*}\right)=0, \mu_{i}^{2}\left(x_{i 2}\right)=1
$$

which impedes that the domains of these functions being overlapped. It can, therefore, be observed that, except for some isolated points,

$$
\mu_{i}^{\mathrm{l}}\left(x_{i}\right)+\mu_{i}^{2}\left(x_{i}\right) \neq \mathbf{1}
$$

and thus, in general, the matrix $\mathrm{X}$ will be of full rank and the method is applicable. This solution can be clearly seen in [1] where the authors find the optimum membership functions minimizing the performance index and reducing the problem to a nonlinear programming one. For this reason, they use the well-known complex method for the minimization. This can obviously be observed in the illustrative examples selected by the authors in [1] where all the identified memberships are non overlapping ones.

\section{PROPOSED APPROACHES}

The restriction of the T-S identification method for the case presented in the previous section does not mean the non-existence of solutions rather than an incentive for their search. As has been seen, the problem comes from the fact that the solution should fulfil:

$$
\nabla J=X^{T} Y-X^{T} X P=0 .
$$

As it was shown above, the columns of the matrix $X$ are linearly dependent and consequently $X^{T} X$ is not an invertible matrix, therefore it is impossible to calculate P through:

$$
P=\left(X^{T} X\right)^{-1} X^{T} Y .
$$

Nevertheless, as the rows of $X^{T}$ are linearly dependent, the independent term in (11) $X^{T} Y$ will have the same dependence among its rows and thereupon the rank of the system matrix will be the same as the rank of the extended matrix with the independent term.

$$
\operatorname{rank}\left(X^{T} X\right)=\operatorname{rank}\left(X^{T} X \mid X^{T} Y\right)
$$

and so the system has a solution. In other words, the system is a compatible indeterminate one, that is, if $\mathrm{P}$ is a solution of (11) and $\mathrm{K}$ belongs to the Kernel of $X^{T} X$

$$
K \in \operatorname{Ker}\left(X^{T} X\right)
$$

then $P^{*}=P+K$ will also be a solution. Therefore, the problem is not the lack of a solution. rather the existence of infinite solutions and the key idea is the ability to find one of them. Several proposals can be made to select a solution. In our case, the aim is to find solutions with lower norm.

\subsection{Minimum norm approach}

The first approach implies the search for the minimum norm solution in an exact and optimum form at the expense of increasing the degree of complexity and computational cost. The problem is stated as follows:

$$
\begin{aligned}
& \text { minimize }\|P\|^{2} \text { with the restriction } \\
& \text { minimize } J=\|Y-X P\|^{2}
\end{aligned}
$$

which can be re-described as follows:

minimize $\|P\|^{2}$ with the restriction

$$
\nabla J=X^{T} Y-X^{T} X P=0
$$

As already stated, the system of equations of restriction is a compatible undetermined one, and therefore, there are linearly dependent restrictions upon others. What is proposed is to eliminate the linear restrictions until obtaining a system with all its restrictions being linearly independent. We obtain a derived system of equations linearly independent

$$
Y_{r}-X_{r} P=0 .
$$

Then, the Lagrange multiplier theorem can be applied, defining a lagrangian function

$$
L=P^{T} P+\lambda^{T}\left(Y_{r}-X_{r} P\right)
$$

that fulfils:

$$
\begin{aligned}
& \nabla_{p} L=2 P^{T}-\lambda^{T} X_{r}=0 \Longrightarrow 2 P-\lambda X_{r}^{T}=0 \\
& \nabla_{\lambda} L=\left(Y_{r}-X_{r} P\right)^{T}=0 \Longrightarrow Y_{r}-X_{r} P=0 .
\end{aligned}
$$

This can also be represented in matrix form:

$$
\left[\begin{array}{cc}
2 I & -X_{r}^{T} \\
X_{r} & 0
\end{array}\right]\left[\begin{array}{l}
P \\
\lambda
\end{array}\right]=\left[\begin{array}{c}
0 \\
Y_{r}
\end{array}\right]
$$


and the solution will be:

$$
\left[\begin{array}{l}
P \\
\lambda
\end{array}\right]=\left[\begin{array}{cc}
2 I & -X_{r}^{T} \\
X_{r} & 0
\end{array}\right]^{-1}\left[\begin{array}{c}
0 \\
Y_{r}
\end{array}\right] .
$$

This possible solution is inconvenient due to the complexity of the algorithm used to calculate the rows of $X^{T} X$, with minimum guarantees, which should be eliminated in order to obtain the matrix $X_{\gamma}$.

\subsection{Weighting parameters approach}

A simple method with reduced computational complexity, based on weighting of parameters, is proposed. The main target is to improve the choice of the performance index and minimize it. It is characterized by extending the objective function by including a weighting $\gamma$ of the norm of $\mathrm{P}$ vector:

$$
\begin{aligned}
J & =\sum_{k=1}^{m}\left(y_{k}-\hat{y}_{k}\right)^{2}+\gamma^{2} \sum_{j} p_{j}^{2} \\
& =\|Y-X P\|^{2}+\gamma^{2}\|P\|^{2} .
\end{aligned}
$$

This can be rewritten as follows:

$$
\begin{aligned}
J & =\|Y-X P\|^{2}+\gamma^{2}\|P\|^{2} \\
& =\left\|\left[\begin{array}{l}
Y \\
0
\end{array}\right]-\left[\begin{array}{c}
X \\
\gamma I
\end{array}\right] P\right\|^{2} \\
& =\left\|Y_{a}-X_{a} P\right\|^{2} .
\end{aligned}
$$

Now the extended matrix $X_{a}$ is of full rank, and the vector $P$ can be computed as:

$$
P=\left(X_{a}^{T} X_{a}\right)^{-1} X_{a}^{T} Y_{a} .
$$

Obviously the solution that minimizes this index is not optimum. However, for a small value of $\gamma$, it will be close to the optimum one and it will be unique as well.

The weighting of $\gamma$ does not need to have a unique value, rather some values can be weighted more than others to choose the most suitable one.

$$
\begin{aligned}
J & =\sum_{k=1}^{m}\left(y_{k}-\hat{y}_{k}\right)^{2}+\sum_{j} \gamma_{j}^{2} p_{j}^{2} \\
& =\|Y-X P\|^{2}+\|\Gamma P\|^{2} \\
& =\left\|\left[\begin{array}{l}
Y \\
0
\end{array}\right]-\left[\begin{array}{l}
X \\
\Gamma
\end{array}\right] P\right\|^{2}
\end{aligned}
$$

where $\Gamma$ is a diagonal matrix formed by the values $\gamma_{j}$ which should necessarliy have a value other than zero to guarantee that the extended matrix is invertible.

\section{EXAMPLES}

In the following examples, we will compare these methods with the one proposed by T-S. Different nonlinear functions will be proposed and the fuzzy models will be obtained assigning an interval $\left[x_{i 1}, x_{i 2}\right]$ for each variable $x_{i}$. In this interval, two fuzzy sets are defined whose membership functions are:

$$
\mu_{i}^{1}=\frac{x_{i}-x_{i 2}}{x_{i 1}-x_{i 2}}, \quad \mu_{i}^{2}=\frac{x_{i}-x_{i 1}}{x_{i 2}-x_{i 1}} .
$$

However, as demonstrated above, these membership functions can not be used directly in the method of T-S, as the resulting matrix $X$ would not be full rank.

In order to compare these methods, we use a factor $0 \leq \alpha \leq 1$, which determines two points within the interval. This means:

$$
\begin{aligned}
& x_{i 1}^{*}=x_{i 2}-\alpha\left(x_{i 2}-x_{i 1}\right) \\
& x_{i 2}^{*}=x_{i 1}+\alpha\left(x_{i 2}-x_{i 1}\right)
\end{aligned}
$$

and so, we define two fuzzy sets whose membership functions are:

$$
\begin{array}{r}
\mu_{i}^{1}(x)= \begin{cases}\frac{x_{i}-x_{i 2}^{*}}{x_{i 1}-x_{i 2}^{*}} & x_{i 1} \leq x \leq x_{i 2}^{*} \\
0 & x_{i 2}^{*} \leq x \leq x_{i 2}\end{cases} \\
\mu_{i}^{2}(x)= \begin{cases}0 & x_{i 1} \leq x \leq x_{i 1}^{*} \\
\frac{x_{i}-x_{i 1}^{*}}{x_{i 2}-x_{i 1}^{*}} & x_{i 1}^{*} \leq x \leq x_{i 2}\end{cases}
\end{array}
$$

which are those used with the direct method of T-S. As a measure of error for comparing these methods, the maximum of the absolute values of the errors is used, which is the same method applied by T-S in [1].

Example 1. Consider the following simple nonlinear system:

$$
\dot{x}=x^{2} .
$$

It is aimed to estimate this system:

$$
y=x^{2} \quad x \in[0,1] \text {. }
$$

Let us suppose that we define in this interval two fuzzy sets with their corresponding membership functions as follows:

$$
\begin{aligned}
& \mu_{1}(x)=1-x \\
& \mu_{2}(x)=x
\end{aligned}
$$


The objective is to calculate the corresponding fuzzy model in an optimum form:

$$
\begin{aligned}
& S^{1}: \text { If } x \text { is } M_{1}^{1} \text { then } y=p_{0}^{1}+p_{1}^{1} x \\
& S^{2}: \text { If } x \text { is } M_{1}^{2} \text { then } y=p_{0}^{2}+p_{1}^{2} x .
\end{aligned}
$$

In order to identify the nonlinear function, we take 20 points uniformly distributed in the interval $[0,1]$. In the minimum norm method, the product $X^{T} X$ is calculated as follows:

$$
X^{T} X=\left[\begin{array}{llll}
6.8421 & 1.5789 & 3.1579 & 1.5789 \\
1.5789 & 0.6333 & 1.5789 & 0.9456 \\
3.1579 & 1.5789 & 6.8421 & 5.2632 \\
1.5789 & 0.9456 & 5.2632 & 4.3175
\end{array}\right]
$$

Its rank is 3 and can be reduced to

$$
X_{\gamma}=\left[\begin{array}{llll}
6.8421 & 1.5789 & 3.1579 & 1.5789 \\
1.5789 & 0.6333 & 1.5789 & 0.9456 \\
3.1579 & 1.5789 & 6.8421 & 5.2632
\end{array}\right] .
$$

The resultant $P$ vector of the parameters in the consequent part is:

$$
P=\left[\begin{array}{c}
0.0000 \\
-0.3333 \\
0.3333 \\
0.6667
\end{array}\right]
$$

This means that the resultant fuzzy rules are:

$$
\begin{aligned}
& S^{1}: \text { If } x \text { is } M_{1}^{1} \text { then } y=-0.3333 x \\
& S^{2}: \text { If } x \text { is } M_{1}^{2} \text { then } y=0.3333+0.6667 .
\end{aligned}
$$

The result in this case is obtained with an error of $7.0031 \mathrm{e}-016$, which is practically zero.

Applying the proposed weighting of parameters approach for various values of $\gamma$ to examine the tradeoff between the accuracy of the identification of the model and the condition number, the following results are obtained:

For $\gamma=0.1$, the obtained fuzzy rules are:

$$
\begin{aligned}
& s^{1}: \text { If } x \text { is } M_{1}^{1} \text { then } y=-0.004-0.3167 x \\
& s^{2}: \text { If } x \text { is } M_{1}^{2} \text { then } y=0.3392+0.6559 x
\end{aligned}
$$

The error between the original system and the identified fuzzy model is with an error of 0.0050 .
For $\gamma=0.01$, the obtained fuzzy rules are:

$$
\begin{aligned}
& s^{1}: \text { If } x \text { is } M_{1}^{1} \text { then } y=-0.3332 x \\
& s^{2}: \text { If } x \text { is } M_{1}^{2} \text { then } y=0.3334+0.66666 x
\end{aligned}
$$

The error between the original system and the identified fuzzy model is with an error of $5.1410 \mathrm{e}-005$.

For $\gamma=0.001$, the obtained fuzzy rules are:

$$
\begin{aligned}
& S^{1}: \text { If } x \text { is } M_{1}^{1} \text { then } y=-0.3333 x \\
& S^{2}: \text { If } x \text { is } M_{1}^{2} \text { then } y=0.3333+0.6667 x .
\end{aligned}
$$

The error between the original system and the identified fuzzy model is with an error of $5.1409 \mathrm{e}-007$.

As can be seen, this is not an optimal solution, but it is obviously very close and presents a good estimation. In addition to that, it can be clearly noticed that as $\gamma$ is reduced, the accuracy of the identified model is tangibly increased though the condition number of the matrix $X_{a}$ is about $3.6608 \mathrm{e}+003$ which is excessively high. In other words, $X_{a}$ is ill-conditioned and this leads to an unreliable results.

In the method of T-S, let us suppose that $\alpha=0.7$. The fuzzy model is:

$$
\begin{aligned}
& S^{1}: \text { If } x \text { is } M_{1}^{1} \text { then } y=-0.0389+0.5238 x \\
& S^{2}: \text { If } x \text { is } M_{1}^{2} \text { then } y=-0.5151+01.4762 x \text {. }
\end{aligned}
$$

The identification error is 0.0201 , which is higher than the one obtained in the proposed two methods. Increasing $\alpha=0.8$, the enror is reduced to 0.0160 , i.e., the identification is improved. With $\alpha=0.9$, the enror becomes 0.0081 , where the identification is again improved. The same occurs for $\alpha=0.95$ where the enror is reduced to 0.0044 . In other words, as the factor $\alpha$ is approaching unity the identification is improved, but it can not reach the optimum which precisely occurs when $\alpha=1$. Since at this value, the matrix $X$ is not of full rank and therefore the matrix $X_{t} X$ is not invertible. Even when $\alpha$ is approaching unity, the condition number of the matrix $\mathrm{X}$ starts increasing which indicates that $X_{t} X$ is approaching the singularity and therefore its inverse is no longer numerically reliable. For instance, when $\alpha=0.999$, the condition number of $X$ is $5.2523 \mathrm{e}+004$ which shows clearly a non reliable result.

Example 2. Consider the following nonlinear system

$$
y=x_{1}^{2}+x_{2}^{2}, \quad x_{1} \in[0,1], \quad x_{2} \in[0,1]
$$


Supposing again that $\gamma=0.1$. Firstly, applying the minimum norm apprach, the fuzzy systems is:

$$
\begin{aligned}
& s^{11} \text { : If } x_{1} \text { is } M_{1}^{1} \text { and } x_{2} \text { is } M_{2}^{1} \\
& \text { then } y=-0.3333 x_{1}-0.3333 x_{2} \\
& s^{12}: \text { If } x_{1} \text { is } M_{1}^{1} \text { and } x_{2} \text { is } M_{2}^{2} \\
& \text { then } y=0.3333-0.2500 x_{1}+0.6667 x_{2} \\
& s^{21}: \text { If } x_{1} \text { is } M_{1}^{2} \text { and } x_{2} \text { is } M_{2}^{1} \\
& \text { then } y=0.3333+0.6667 x_{1}-0.2500 x_{2} \\
& s^{22}: \text { If } x_{1} \text { is } M_{1}^{2} \text { and } x_{2} \text { is } M_{2}^{2} \\
& \text { then } y=0.5000+0.7500 x_{1}+0.7500 x_{2}
\end{aligned}
$$

with an error of $7.1497 \mathrm{e}-015$, i.e. approximately zero. Applying the weighting of parameters approach, the local dynamics of the fuzzy system become:

$$
\begin{aligned}
& S^{11} \text { : If } x_{1} \text { is } M_{1}^{1} \text { and } x_{2} \text { is } M_{2}^{1} \\
& \text { then } y=-0.0002-0.3330 x_{1}-0.3330 x_{2} \\
& S^{12}: \text { If } x_{1} \text { is } M_{1}^{1} \text { and } x_{2} \text { is } M_{2}^{2} \\
& \quad \text { then } y=0.3334-0.2497 x_{1}+0.6664 x_{2} \\
& s^{21}: \text { If } x_{1} \text { is } M_{1}^{2} \text { and } x_{2} \text { is } M_{2}^{1} \\
& \quad \text { then } y=0.3334+0.6664 x_{1}-0.2498 x_{2} \\
& s^{22}: \text { If } x_{1} \text { is } M_{1}^{2} \text { and } x_{2} \text { is } M_{2}^{2} \\
& \text { then } y=0.5002+0.7498 x_{1}+0.7498 x_{2}
\end{aligned}
$$

The identification model is obtained with an error of 4.6771e-005. Applying the method presented by T-S, with $\alpha=0.9$ for $x_{1}$ and $x_{2}$, the resultant fuzzy model is:

$$
\begin{aligned}
& s^{11}: \text { If } x_{1} \text { is } M_{1}^{1} \text { and } x_{2} \text { is } M_{2}^{1} \\
& \text { then } y=-0.0287+0.5473 x_{1}-4.6928 x_{2} \\
& s^{12}: \text { If } x_{1} \text { is } M_{1}^{1} \text { and } x_{2} \text { is } M_{2}^{2} \\
& \text { then } y=4.6843-0.5473 x_{1}-3.2235 x_{2} \\
& s^{21}: \text { If } x_{1} \text { is } M_{1}^{2} \text { and } x_{2} \text { is } M_{2}^{1} \\
& \text { then } y=-0.4814+1.4527 x_{1}-5.5301 x_{2} \\
& s^{22}: \text { If } x_{1} \text { is } M_{1}^{2} \text { and } x_{2} \text { is } M_{2}^{2} \\
& \text { then } y=5.1964+4.4527 x_{1}-0.7498 x_{2}
\end{aligned}
$$

The resultant error is 0.0515 , and the condition number for the matrix $X$ is $3.4381 \mathrm{e}+015$ which is very close to singularity.
Example 3. Let us analyze the following non linear system:

$$
y=x_{1}^{3}+x_{2}^{3} x_{1} \in[0,1] x_{2} \in[0,1] .
$$

Supposing again that $\gamma=0.1$. Firstly, applying the minimum norm apprach, the fuzzy systems is:

$$
\begin{aligned}
& s^{11}: \text { If } x_{1} \text { is } M_{1}^{1} \text { and } x_{2} \text { is } M_{2}^{1} \\
& \text { then } y=-0.0016+0.9510 x_{1}-0.6985 x_{2} \\
& s^{12}: \text { If } x_{1} \text { is } M_{1}^{1} \text { and } x_{2} \text { is } M_{2}^{2} \\
& \text { then } y=0.1030+0.9510 x_{1}+0.8015 x_{2} \\
& s^{21} \text { : If } x_{1} \text { is } M_{1}^{2} \text { and } x_{2} \text { is } M_{2}^{1} \\
& \text { then } y=-1.2998+2.3090 x_{1}-1.1312 x_{2} \\
& s^{22}: \text { If } x_{1} \text { is } M_{1}^{2} \text { and } x_{2} \text { is } M_{2}^{2} \\
& \text { then } y=-0.7625+2.3090 x_{1}+0.3688 x_{2}
\end{aligned}
$$

with an identification error of $2.14 \mathrm{e}-02$. Applying, the weighting of parameters approach, for $\gamma=0.01$, the fuzzy model is approximately the same as before:

$$
\begin{aligned}
& S^{11} \text { : If } x_{1} \text { is } M_{1}^{1} \text { and } x_{2} \text { is } M_{2}^{1} \\
& \text { then } y=-0.0015+0.9503 x_{1}-0.6985 x_{2} \\
& s^{12}: \text { If } x_{1} \text { is } M_{1}^{1} \text { and } x_{2} \text { is } M_{2}^{2} \\
& \text { then } y=0.1030+0.9505 x_{1}+0.8015 x_{2} \\
& S^{21}: \text { If } x_{1} \text { is } M_{1}^{2} \text { and } x_{2} \text { is } M_{2}^{1} \\
& \text { then } y=-1.2991+2.308 x_{1}-1.1311 x_{2} \\
& S^{22}: \text { If } x_{1} \text { is } M_{1}^{2} \text { and } x_{2} \text { is } M_{2}^{2} \\
& \text { then } y=-0.7622+2.3086 x_{1}+0.3689 x_{2}
\end{aligned}
$$

with the same identification error of 0.0214 . But in the T-S method, using $\alpha=0.8$, which is the value that after several attempts provides better results, we get:

$$
\begin{aligned}
& s^{11} \text { : If } x_{1} \text { is } M_{1}^{1} \text { and } x_{2} \text { is } M_{2}^{1} \\
& \text { then } y=-0.0085+0.5910 x_{1}-1.05415 x_{2} \\
& s^{12} \text { : If } x_{1} \text { is } M_{1}^{1} \text { and } x_{2} \text { is } M_{2}^{2} \\
& \text { then } y=0.4578+0.5910 x_{1}+0.4273 x_{2} \\
& s^{21} \text { : If } x_{1} \text { is } M_{1}^{2} \text { and } x_{2} \text { is } M_{2}^{1} \\
& \text { then } y=-0.9482+1.9274 x_{1}-1.3607 x_{2} \\
& S^{22} \text { : If } x_{1} \text { is } M_{1}^{2} \text { and } x_{2} \text { is } M_{2}^{2} \\
& \text { then } y=-0.1880+1.9274 x_{1}+0.1465 x_{2}
\end{aligned}
$$


with an identification error of 0.0307 . In this case, with $\alpha=0.9$ the results becomes worse because the condition number for the matrix $X$ is $3.4381 \mathrm{e}+015$ which is the same as that in the previous case, where the matrix $X$ does not depend upon the function rather on the interval.

Example 4. In this example, we will analyze the control of $\mathrm{pH}$ which is a very important problem in several processes. especially in effluent wastewater treatment. We have chosen this example for its importance being a highly nonlinear system. Another reason for choosing this example is because it is the same one analyzed by $\mathrm{T}-\mathrm{S}$, so that we can compare the results obtained applying our proposed methods with the results obtained by the method developed in T-S. The process consists of a tank of a constant volume $\mathrm{V}$ which receives a flow of waste water $q_{v}$ which is supposed to be constant with a variable $p H_{v}$. It is supposed to be an agitated tank and therefore the output concentrations are the same as those of the tank. To control the output of $\mathrm{pH}_{s}$, a flow of acid concentration $q_{a}$ can be injected with constant $\left[\mathrm{H}^{+}\right]_{a}$ and/or a flow base $q_{b}$ with constant concentration $\left[\mathrm{OH}^{-}\right]_{b}$. These concentrations lead to highly nonlinear algebraic equations. In order to explain the plant equations, it should be taken into consideration that in an aqueous solution, the following conditions should be verified:

$$
\begin{aligned}
p H & =-\log _{10}\left[H^{+}\right] \\
p O H & =-\log _{10}\left[O H^{-}\right] \\
p H+p O H & =14 \Rightarrow\left[H^{+}\right]\left[O H^{-}\right]=10^{-14} .
\end{aligned}
$$

If the waste water volume has $p H_{v}$ then it is verified that.

$$
\begin{aligned}
{\left[H^{+}\right]_{v} } & =10^{-p H} \\
{\left[O H^{-}\right]_{v} } & =\frac{10^{-14}}{\left[H^{+}\right]_{v}} .
\end{aligned}
$$

As regards to the acid and base volumes, they can not be considered as aqueous solutions and their concentrations do not have this effect. Using the notations $\left[\mathrm{H}^{+}\right]_{k}$ and $[\mathrm{OH}-]_{k}$ for the concentrations in the tank at the instant $\mathbf{k}$, a discrete model is suggested with a sampling time of $\mathrm{T}$. The equilibrium mass equations are:

$$
\begin{aligned}
V\left[H^{+}\right]_{k+1}^{+}= & V\left[H^{+}\right]_{k}+T q_{v}\left[H^{+}\right]_{v k} \\
& +T q_{a}\left[H^{+}\right]_{a}-T q_{s}\left[H^{+}\right]_{k} \\
V\left[O H^{-}\right]_{k+1}^{+}= & V\left[O H^{-}\right]_{k}+T q_{v}\left[O H^{-}\right]_{v k} \\
& +T q_{b}\left[O H^{-}\right]_{b}-T q_{s}\left[O H^{-}\right]_{k}
\end{aligned}
$$

Generally, the values $\left[\mathrm{H}^{+}\right]_{k+1}^{+}$and $\left[\mathrm{OH}^{-}\right]_{k+1}^{+}$do not fulfil the restriction (28) with what is supposed that, instantly, a portion of these concentrations are combined to form water. For its calculation we use the following equation:

$$
\left(\left[\mathrm{H}^{+}\right]_{k+1}^{+}-x\right)\left(\left[\mathrm{OH}^{-}\right]_{k+1}^{+}-x\right)=10^{-14}
$$

and finally

$$
\begin{gathered}
{\left[\mathrm{H}^{+}\right]_{k+1}=\left[\mathrm{H}^{+}\right]_{k+1}^{+}-x} \\
{\left[O \mathrm{H}^{-}\right]_{k+1}=\left[O \mathrm{OH}^{-}\right]_{k+1}^{+}-x}
\end{gathered}
$$

The output volume, which is considered constant should verify:

$$
q_{s}=q_{v}+q_{a}+q_{b}
$$

The following numerical values are used for the constants:

$$
\begin{aligned}
V & =5000 l \\
q_{v} & =1 \frac{l}{s} \\
{\left[H^{+}\right]_{a} } & =0.01 \frac{\mathrm{m}}{l} \\
{\left[O H^{-}\right]_{b} } & =0.01 \frac{\mathrm{m}}{l} \\
T & =0.1 s .
\end{aligned}
$$

It can be assumed that the acid and base volumes do not exist simultaneously under normal conditions of the plant, and therefore the control variable is a volume of reactive value $q_{r}$ which should verify, for example,

$$
\begin{aligned}
& q_{a}= \begin{cases}q_{r} & \text { if } q_{r} \geq 0 \\
0 & \text { if } q_{r} \geq 0\end{cases} \\
& q_{b}= \begin{cases}0 & \text { if } q_{r} \geq 0 \\
-q_{r} & \text { if } q_{r} \geq 0\end{cases}
\end{aligned} .
$$

The problem therefore is the modelling of:

$$
p H_{k+1}=f\left(p H_{k}, p H_{v k}, q_{r k}\right) .
$$

The experiment was to introduce a flow rate of discharge with variable $\mathrm{pH}$ modelled through a random variable with a function of normal distribution $\mathrm{N}(7,0.7)$ changing every 120 seconds. The reactive flow rate has been modelled with $\mathrm{N}(0,0.001)$ changing every $110 \mathrm{~s}$. The $\mathrm{pH}$ of the output has been recorded every $10 \mathrm{~s}$ (see Figs 2-4). For the simplification of the notation, we use:

$$
\begin{aligned}
& x_{1}=p H_{k} \\
& x_{2}=p H_{v k} \\
& x_{3}=q_{r k}
\end{aligned}
$$




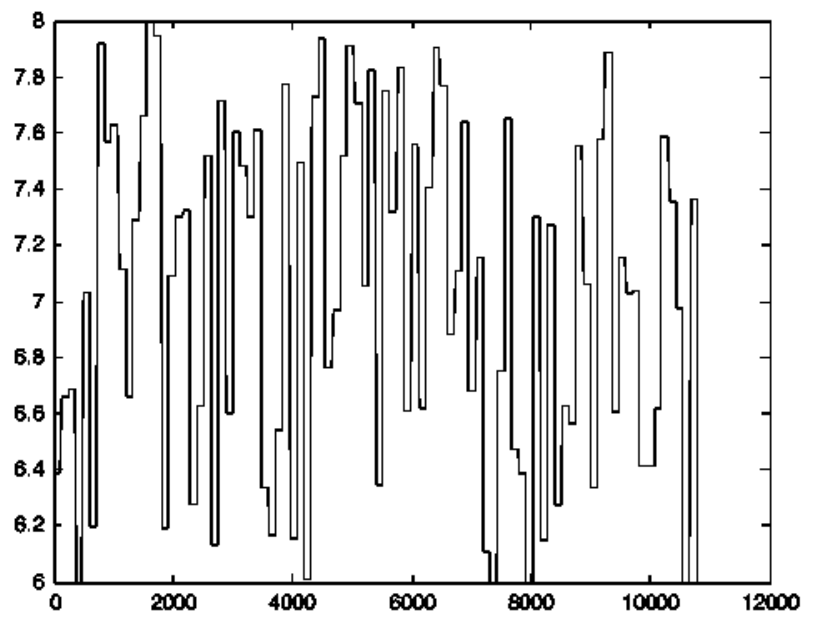

Fig. 2. $\mathrm{pHv}$ variable.

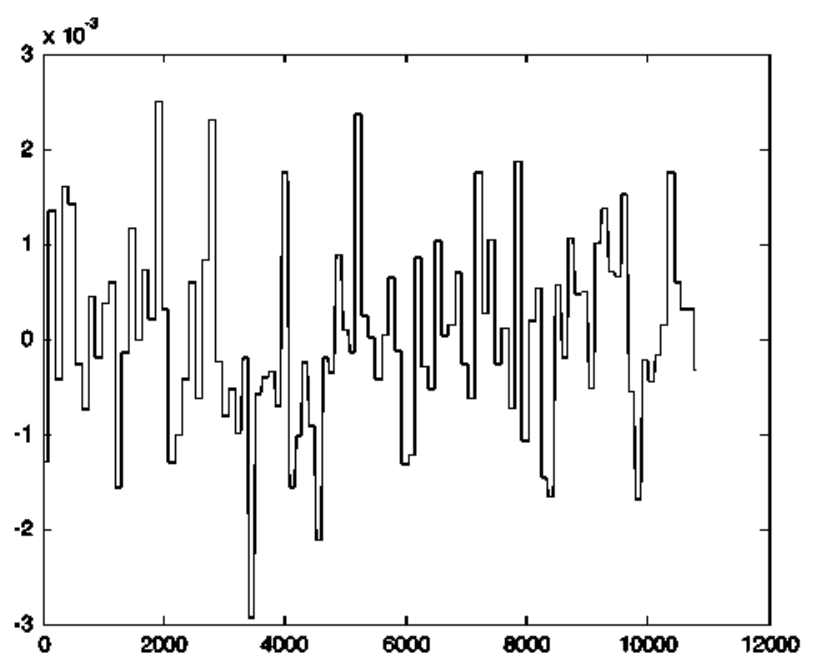

Fig. 3. Reactive flow rate.

Now we take as intervals of identification, those which lie between the minimum and maximum value for each of the variables. Applying the minimum noim approach.

$s^{111}$ :If $x_{1}$ is $M_{1}^{1}$ and $x_{2}$ is $M_{2}^{1}$ and $x_{3}$ is $M_{3}^{1}$ then

$$
y=0.0724+0.7133 x_{1}+0.2739 x_{2}-0.0104 x_{3}
$$

$s^{112}$ :If $x_{1}$ is $M_{1}^{1}$ and $x_{2}$ is $M_{2}^{1}$ and $x_{3}$ is $M_{3}^{2}$ then

$$
y=0.1317+0.4489 x_{1}+0.5232 x_{2}+0.0105 x_{3}
$$

$s^{121}$ : If $x_{1}$ is $M_{1}^{1}$ and $x_{2}$ is $M_{2}^{2}$ and $x_{3}$ is $M_{3}^{1}$ then

$$
y=0.0220+0.5486 x_{1}+0.3366 x_{2}-0.0058 x_{3}
$$

$S^{122}$ : If $x_{1}$ is $M_{1}^{1}$ and $x_{2}$ is $M_{2}^{2}$ and $x_{3}$ is $M_{3}^{2}$ then

$$
y=0.0058+0.3510 x_{1}+0.4676 x_{2}+0.4676 x_{3}
$$

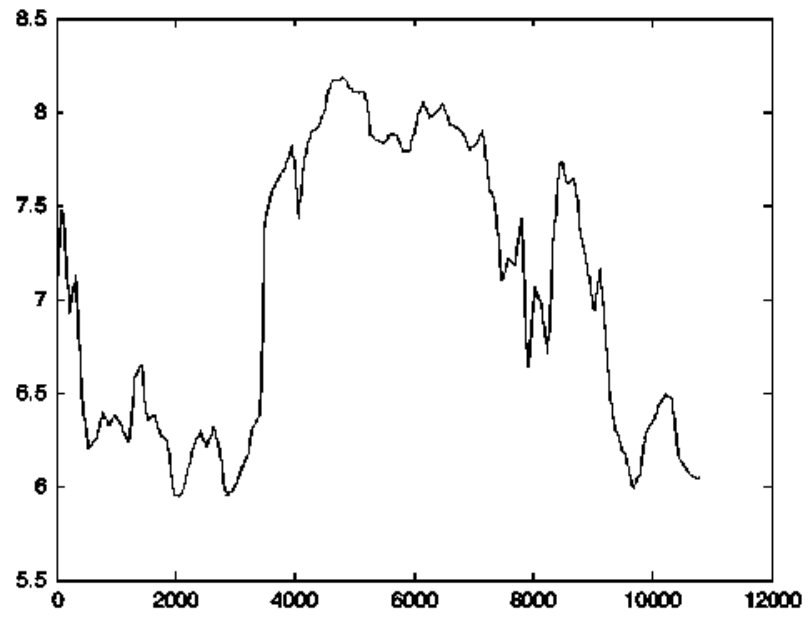

Fig. 4. $\mathrm{pHs}$ variable.

$S^{211}$ :If $x_{1}$ is $M_{1}^{2}$ and $x_{2}$ is $M_{2}^{1}$ and $x_{3}$ is $M_{3}^{1}$ then

$$
y=0.0946+0.4904 x_{1}+0.6725 x_{2}-0.0050 x_{3}
$$

$S^{212}$ : If $x_{1}$ is $M_{1}^{2}$ and $x_{2}$ is $M_{2}^{1}$ and $x_{3}$ is $M_{3}^{2}$ then

$$
y=0.0401+0.6616 x_{1}+0.4613 x_{2}+0.0049 x_{3}
$$

$S^{221}$ : If $x_{1}$ is $M_{1}^{2}$ and $x_{2}$ is $M_{2}^{2}$ and $x_{3}$ is $M_{3}^{1}$ then

$$
y=0.0942+0.3529 x_{1}+0.6483 x_{2}-0.0023 x_{3}
$$

$s^{222}$ : If $x_{1}$ is $M_{1}^{2}$ and $x_{2}$ is $M_{2}^{2}$ and $x_{3}$ is $M_{3}^{2}$ then

$$
y=-0.0876+0.5213 x_{1}+0.4797 x_{2}+0.0023 x_{3}
$$

with an error of 0.0254 . Applying the weighting of parameters approach, for $\gamma=0.1$,

$s^{111}$ :If $x_{1}$ is $M_{1}^{1}$ and $x_{2}$ is $M_{2}^{1}$ and $x_{3}$ is $M_{3}^{1}$ then

$$
y=-0.0137+0.6920 x_{1}+0.3084 x_{2}-0.0017 x_{3}
$$

$S^{112}$ :If $x_{1}$ is $M_{1}^{1}$ and $x_{2}$ is $M_{2}^{1}$ and $x_{3}$ is $M_{3}^{2}$ then

$$
y=0.1972+0.4718 x_{1}+0.4903 x_{2}+0.0022 x_{3}
$$

$s^{121}$ :If $x_{1}$ is $M_{1}^{1}$ and $x_{2}$ is $M_{2}^{2}$ and $x_{3}$ is $M_{3}^{1}$ then

$$
y=0.0901+0.5441 x_{1}+0.3302 x_{2}-0.0037 x_{3}
$$

$S^{122}$ : If $x_{1}$ is $M_{1}^{1}$ and $x_{2}$ is $M_{2}^{2}$ and $x_{3}$ is $M_{3}^{2}$ then

$$
y=-0.0268+0.3577 x_{1}+0.4790 x_{2}+0033 x_{3}
$$

$s^{211}$ :If $x_{1}$ is $M_{1}^{2}$ and $x_{2}$ is $M_{2}^{1}$ and $x_{3}$ is $M_{3}^{1}$ then

$$
y=0.1537+0.4842 x_{1}+0.6715 x_{2}-0.0034 x_{3}
$$

$s^{212}$ : If $x_{1}$ is $M_{1}^{2}$ and $x_{2}$ is $M_{2}^{1}$ and $x_{3}$ is $M_{3}^{2}$ then

$$
y=-0.0043+0.6652 x_{1}+0.4630 x_{2}+0.0029 x_{3}
$$




$$
\begin{gathered}
S^{211} \text { :If } x_{1} \text { is } M_{1}^{2} \text { and } x_{2} \text { is } M_{2}^{1} \text { and } x_{3} \text { is } M_{3}^{1} \text { then } \\
y=0.1537+0.4842 x_{1}+0.6715 x_{2}-0.0034 x_{3} \\
S^{212} \text { :If } x_{1} \text { is } M_{1}^{2} \text { and } x_{2} \text { is } M_{2}^{1} \text { and } x_{3} \text { is } M_{3}^{2} \text { then } \\
y=-0.0043+0.6652 x_{1}+0.4630 x_{2}+0.0029 x_{3} \\
S^{221}: \text { If } x_{1} \text { is } M_{1}^{2} \text { and } x_{2} \text { is } M_{2}^{2} \text { and } x_{3} \text { is } M_{3}^{1} \text { then } \\
y=0.0473+0.3778 x_{1}+0.6288 x_{2}-0.0054 x_{3} \\
S^{222}: \text { If } x_{1} \text { is } M_{1}^{2} \text { and } x_{2} \text { is } M_{2}^{2} \text { and } x_{3} \text { is } M_{3}^{2} \text { then } \\
y=0.1238+0.4957 x_{1}+0.5014 x_{2}+0.0056 x_{3}
\end{gathered}
$$

with an error of 0.0271 . Using the method developed in [1] by $\mathrm{T}-\mathrm{S}$ with $\alpha=0.7$, the error is 3.0089 , with $\alpha=0.8$ the error is 1.9480 and finally the best results are obtained with $\alpha=0.9$ as follows:

$$
\begin{aligned}
& S^{111} \text { :If } x_{1} \text { is } M_{1}^{1} \text { and } x_{2} \text { is } M_{2}^{1} \text { and } x_{3} \text { is } M_{3}^{1} \text { then } \\
& y=-15.773-1.7710 x_{1}+5.6135 x_{2}-156.60 x_{3} \\
& s^{112} \text { :If } x_{1} \text { is } M_{1}^{1} \text { and } x_{2} \text { is } M_{2}^{1} \text { and } x_{3} \text { is } M_{3}^{2} \text { then } \\
& y=-59.866+11.174 x_{1}-0.0595 x_{2}-409.41 x_{3} \\
& s^{121} \text { :If } x_{1} \text { is } M_{1}^{1} \text { and } x_{2} \text { is } M_{2}^{2} \text { and } x_{3} \text { is } M_{3}^{1} \text { then } \\
& y=-25.268-1.1196 x_{1}+5.0798 x_{2}+253.08 x_{3} \\
& s^{122} \text { :If } x_{1} \text { is } M_{1}^{1} \text { and } x_{2} \text { is } M_{2}^{2} \text { and } x_{3} \text { is } M_{3}^{2} \text { then } \\
& y=35.291-1.8842 x_{1}-2.0076 x_{2}-412.26 x_{3} \\
& S^{211} \text { :If } x_{1} \text { is } M_{1}^{2} \text { and } x_{2} \text { is } M_{2}^{1} \text { and } x_{3} \text { is } M_{3}^{1} \text { then } \\
& y=47.183-3.2258 x_{1}-0.7815 x_{2}+2749.9 x_{3} \\
& S^{212} \text { :If } x_{1} \text { is } M_{1}^{2} \text { and } x_{2} \text { is } M_{2}^{1} \text { and } x_{3} \text { is } M_{3}^{2} \text { then } \\
& y=-106.24+10.960 x_{1}+3.9854 x_{2}+2724.8 x_{3} \\
& s^{221} \text { : If } x_{1} \text { is } M_{1}^{2} \text { and } x_{2} \text { is } M_{2}^{2} \text { and } x_{3} \text { is } M_{3}^{1} \text { then } \\
& y=39.784-1.9902 x_{1}-3.1530 x_{2}-4678.6 x_{3} \\
& s^{222} \text { :If } x_{1} \text { is } M_{1}^{2} \text { and } x_{2} \text { is } M_{2}^{2} \text { and } x_{3} \text { is } M_{3}^{2} \text { then } \\
& y=20.677-4.44981 x_{1}+4.5422 x_{2}-4943.7 x_{3}
\end{aligned}
$$

with an error of 0.7788 . Choosing $\alpha=0.95$, the error is 0.3449 with a condition number of the matrix $\mathrm{X}$ of $1.8242 \mathrm{e}+006$, i.e., its columns are practically linearly dependent and the results are not reliable.

\section{CONCLUSIONS}

Two new optimization methods have been developed to improve the local and global approximation and modelling capability of the Takagi-Sugeno (T-S) identification methodology model. The main problem encountered is that the T-S identification method can not be applied when the membership functions are overlapped by pairs. This restricts the application of the T-S identification method because this type of membership function has been widely used during the last two decades in fuzzy control applications. Two solutions have been proposed to reduce the error between the original system and the identified one. The first uses the minimum norm approach which implies the search for an exact and optimum solution at the expense of increasing the degree of complexity and computational cost. The second is a simple and less computational method, based on weighting of parameters. The main target is to improve the choice of the performance index and minimize it. The results obtained have shown tangible improvement compared to the solution offered by T-S. Several illustrative examples have been presented to evaluate the validity and performance of the proposed methods and the high accuracy obtained in approximating nonlinear systems locally and globally in comparison with the original T-S model. Simulation results indicate the potential, simplicity and generality of the algorithm. One of the proposed examples which treats the $\mathrm{PH}$ control problem is the same one that was analyzed by T-S. The results obtained by applying the proposed methods have demonstrated better results in comparison with the original T-S.

\section{REFERENCES}

1. Takagi, T. and M. Sugeno, "Fuzzy identification of systems and its applications to modeling and control," IEEE Trans. Syst. Man Cybern., Vol. SMC15, No. 1, pp. 116-132 (1985).

2. Juang. C. F." "An automatic building approach to special T-S fuzzy network for unknown plant modeling and stable control," Asian J. Control, Vol. 5, No. 2, pp. 176-186 (2003).

3. Mencattini, A., M. Salmeri, and A. Salsano, "Sufficient conditions to impose derivative constraints on MISO Takagi-Sugeno fuzzy logic systems," IEEE Trans. Fuzzy Syst. Vol. 13, No. 4 (2005).

4. Baranyi, P., "SVD-based reduction to MISO TS models," IEEE Trans. Ind. Electron., Vol. 51, No. 1, pp. 232-242 (2003).

5. Hou, Y.. J. M. Zurada, W. Karwowski, W. S. Marras, and $\mathrm{K}$. Davis, "Identification of key variables using 
fuzzy average with fuzzy cluster distribution," IEEE Trans. Fuzzy Syst., Vol. 15, No. 4, pp. 673-685 (2007).

6. Gang, F., "A survey on analysis and design of model-based fuzzy control systems," IEEE Trans. Fuzzy Syst., Vol. 14, No. 5, pp. 676-697 (2006).

7. Guerra, T. M. and L. Vermeiren, "LMI-based relaxed nonquadratic stabilization conditions for nonlinear systems in the Takagi-Sugeno's form," Automatica, Vol. 40, pp. 823-829 (2004).

8. Hseng, T., S. Li, and S.-H. Tsai, "T-S fuzzy bilinear model and fuzzy controller design for a class of nonlinear systems," IEEE Trans. Fuzzy Syst., Vol. 15, No. 3, pp. 494-506 (2007).

9. Jiang, $X$. and Q.-L. Han, "Robust $H$ control for uncertain Takagi-Sugeno fuzzy systems with interval time-varying delay," IEEE Trans. Fuzzy Syst., Vol. 15, No. 2, pp. 321-331 (2007).

10. Chen, B., X. Liu, and S. Tong, "Adaptive fuzzy output tracking control of MIMO nonlinear uncertain systems," IEEE Trans. Fuzzy Syst, Vol. 15. No. 2. pp. 287-300 (2007).

11. Jae-Hun, K., C.-H. Hyun, E. Kim, and M. Park, "New adaptive synchronization of uncertain chaotic systems based on T-S fuzzy model," IEEE Trans. Fuzzy Syst., Vol. 15, No. 3, pp. 359-369 (2007).

12. Zeng, K., N. Y. Zhang, and W. L. Xu, "A comparative study on sufficient conditions for Takagi-Sugeno fuzzy systems as universal approximators," IEEE Trans. Fuzzy Syst., Vol. 8, No. 6, pp. 773-780 (2000).

13. Johansen, T., A. R. Shorten, and R. Murray-Smith, "On the interpretation and identification of dynamic Takagi-Sugeno models." IEEE Trans. Fuzzy Syst., Vol. 8, No. 3, pp. 297-313 (2000).

14. Lian, K.-Y., C.-H. Su, and C.-S. Huang, "Performance enhancement for T-S fuzzy control using neural networks," IEEE Trans. Fuzzy Syst. Vol. 14, No. 5, pp. 619-627 (2006).

15. Kóczy, L. T. and K. Hirota, "Size reduction by interpolation in fuzzy rule bases," IEEE Trans. Syst. Man Cybern. Part B, Vol. 27, No. 1, pp. 14-25 (1997).

16. Tikk, D., P. Baranyi, and R. J. Patton, "Polytopic and TS models are nowere dense in the approximation model space, Proc. IEEE Int. Conf. Syst. Man Cybern. Hammamet, Tunisia (2002).

17. Baranyi, P., P. Korondi, R. J. Patton, and H. Hashimoto. "Global asymptotic stabilisation of the prototypical aeroelastic wing section via TP model transformation." Asian J. Control, Vol. 7, No. 2, pp. 99-111 (2005).
18. Tikk, D., P. Baranyi, and R. J. Patton, "Approximation properties of TP forms and its consequences to TPDC design framework," Asian J. Control, Vol. 9, No. 3, pp. 221-231 (2007).

19. Szeidl, L. and P. Varlaki, "HOSVD based canonical form for polytopic models of dynamic systems." $J$. Adv. Comp. Intell. Intell. Inform., Vol. 13. No. 1, pp. 52-60 (2009).

20. Yam, Y. P. Baranyi, and C.-T. Yang. "Reduction of fuzzy rule base via singular value decomposition." IEEE Trans. Fuzzy Syst., Vol. 7, No. 2, pp. 120-132 (1999).

21. Matía, F., A. Jiménez, and B. Al-Hadithi, "An affine model with decoupled dynamics," Proc. 12th Int. Conf. Inf. Process. Manage. Uncertainty Knowl.Based Syst. Malaga, Spain, pp. 713-720 (2008).

22. Cao, S. G.. N. W. Rees, and G. Feng. "Analysis and design for a class of complex control systems-Part I: fuzzy modeling and identification." Automatica, Vol. 33, pp. 1017-1028 (1997).

23. Mollov, S., R. Babuska, J. Abonyi, and $H$. B. Verbruggen, "Effective optimization for fuzzy model predictive control," IEEE Trans. Fuzzy Syst., Vol. 12, No. 5, pp. 661-675 (2004).

24. Skrjanc, I., S. Blazic, and O. Agamennoni, "Interval fuzzy model identification using $l_{\infty}$-norm," IEEE Trans. Fuzzy Syst., Vol. 13, No. 5, pp. 561-568. (2005).

25. Wang, L. X. and J. M. Mendel, "Generating fuzzy rules by learning from examples," IEEE Trans. Syst. Man Cybem., Vol. 22, pp. 1414-1427 (1992).

26. Klawonn, F. and R. Kruse, "Constructing a fuzzy controller from data," Fuzzy Sets Syst., Vol. 85, No. 2, pp. 177-193 (1997).

27. Hong, T. P. and C. Y. Lee, "Induction of fuzzy rules and membership functions from training examples," Fuzzy Sets Syst., Vol. 84, No. 1, pp. 33-47 (1996).

28. Kim. J., Y. Suga, and S. Won, "A new approach to fuzzy modeling of nonlinear dynamic systems with noise: relevance vector learning mechanism," IEEE Trans. Fuzzy Syst. Vol. 14, No. 2, pp. 222-231 (2006).

29. Yam, Y., "Fuzzy approximation via grid point sampling and singular value decomposition," IEEE Trans. Syst. Man Cybern. Part B, Vol. 27, No. 6, pp. 933-951 (1997).

30. Baranyi, P., P. Korondi, R. J. Patton, and H. Hashimoto, "Trade-off between approximation accuracy and complexity for T-S fuzzy models," Asian J. Control, Vol. 6. No. 1. pp. 21-33 (2004).

31. Tikk, D. and P. Baranyi. "Comprehensive analysis of a new fuzzy rule interpolation method," IEEE Trans. Fuzzy Syst., Vol. 8, No. 3, pp. 281-296 (2000). 
32. Baranyi, P., L. T. Kóczy, and Tamás D. Gedeon, "A generalized concept for fuzzy rule interpolation," IEEE Trans. Fuzzy Syst., Vol. 12, No. 6, pp. 820-837 (2004).

33. Yam, Y., M. L. Wong, and P. Baranyi, "Interpolation with function space representation of membership functions," IEEE Trans. Fuzzy Syst., Vol. 14, No. 3, pp. 398-411 (2006).

34. Yam, Y. and L. T. Kóczy, "Cartesian representation for fuzzy interpolation, Proc. 37th Conf. Decis. Control, Tampa, FL, pp. 2936-2937 (1998).

35. Yam, Y. and L. T. Kóczy, "Representing membership functions as points in high dimensional spaces for fuzzy interpolation and extrapolation," IEEE Trans. Fuzzy Syst., Vol. 8, No. 6, pp. 761-772 (2000).

36. Wang, L., Adaptive Fuzzy Systems and Control: Design and Stability Analysis, Prentice-Hall, Englewood Cliffs, NJ, pp. 27-28 (1994).

37. Wang, L. X. and J. M. Mendel, "Design and analysis of fuzzy identifiers of nonlinear dynamic systems," IEEE Trans. Autom. Control, Vol. 40, pp. 11-23 (1995).

38. Langari, R. and L. Wang, "Complex systems modeling via fuzzy logic," IEEE Trans. Syst. Man Cybern. Part B, Vol. 26, pp. 100-106 (1996).

39. Nozaki, K., H. Ishibuchi, and H. Tanaka, "A simple but powerful heuristic method for generating fuzzy rules from numerical data," Fuzzy Sets Syst., Vol. 86, No. 3, pp. 251-270 (1997).

40. Kumar, M., R. Stoll, and N. Stoll, "A minmax approach to fuzzy clustering, estimation, and identification," IEEE Trans. Fuzzy Syst., Vol. 14, No. 2, pp. 248-262 (2006).

41. Chen, W. and M. Saif, "A novel fuzzy system with dynamic rule base," IEEE Trans. Fuzzy Syst., Vol. 13, No. 5, pp. 569-582 (2005).

42. Jiménez, A., B. M. Al-Hadithi, and F. Matía, "An optimal T-S model for the estimation and identification of nonlinear functions," WSEAS Trans. Syst. Control, Vol. 3, No. 10, pp. 897-906 (2008).

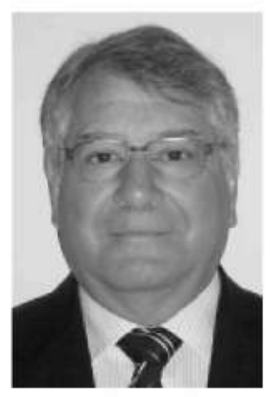

Agustín Jiménez was born in Toledo, Spain, in 1952. He received his B.S. degree in Industrial Engineering and his $\mathrm{Ph} . \mathrm{D}$. degree in 1974 and 1978, respectively, from the Universidad Politécnica de Madrid, Madrid, Spain. He is currently a Professor at the Universidad Politécnica de Madrid, where he is also with the Intelligent Control Research Group. His main teaching and research activities have been related to the development and application of advanced technologies to process control, particularly the integration of artificial intelligence technologies with conventional control applications. He has published different papers and books. His experience in R\&D projects is very wide, having taken part in numerous international projects, particularly financed by the European Community, in many projects of the Spanish National Plan, and in many projects of technological transfer to industry.

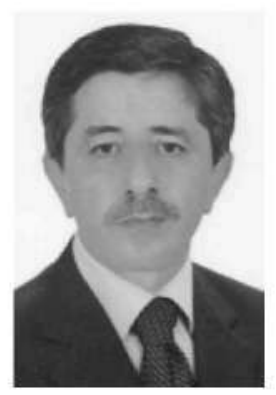

Basil M. Al-Hadithi received his B.Sc. degree in Control and System Engineering and his M.Sc. Engineering degree in Control and Instrumentation both from the University of Technology, Baghdad, Iraq, in 1983 and 1988 respectively. He received his $\mathrm{Ph} . \mathrm{D}$. degree in Process Control and Artificial Intelligence from the Universidad Politécnica de Madrid, in 2002.

He was an Assistant Professor at university of Technology from 1990-1995, Baghdad, Iraq. From 1999-2009 he was an Associate Professor at the Electronic and Systems Department at Universidad Alfonso X El Sabio, Madrid, Spain. Since 2009 he has been with the Universidad Politécnica de Madrid where he is engaged in teaching and research in the area of control theory and control engineering. His research interests include variable structure control, adaptive control and fuzzy modelling and control with applications. He is the author and co-author of various books, journal and conference articles.

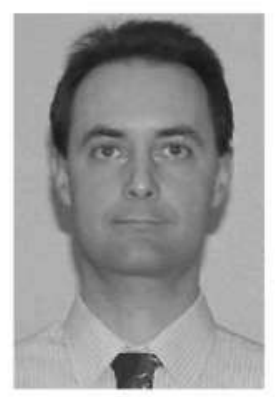

Fernando Matía is a Full Professor at the Universidad Politécnica de Madrid since 2010, has been Associate Professor since 1997. He received his Ph.D. from that University in 1994, being engaged in Control Engineering since 1988, and in Robotics since 1994. His main R\&D activities are fuzzy control and mobile robotics. $\mathrm{He}$ has experienced in eight European projects and 17 national research and industrial projects related with the previous topics. $\mathrm{He}$ is the author of three books, 16 book chapters, 26 articles in journals, and 75 papers in conferences, being reviewer of 12 international journals. He has also been Deputy Vice-Director 
of Studies, Vice-Director of Quality Management and Vice-Director of Studies of the High School of Industrial Engineering at Universidad Politécnica de Madrid.

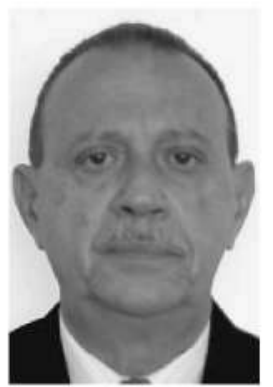

Rodolfo Haber graduated in Electrical Engineering from the University of Oriente (UO, Santiago de Cuba, Cuba) in 1964, and obtained his $\mathrm{Ph} . \mathrm{D}$. degree in Technical Cybernetics at Prague Technical University (CVUT),
Czech Republic, in 1976. Between 1966 and 2009, he is a Full Professor of Automatic Control Theory (and related subjects), at the Automatic Control Dept., Electrical Engineering Faculty, UO. During 1993-94 he joined the Industrial Automatics Institute (CSIC), Madrid, Spain, as an Invited Researcher. During 200809, he joined the Polytechnic University of Madrid, Spain, as an Invited Professor. He is an Honorary Member of the Cuban National permanent Commission for $\mathrm{Ph} . \mathrm{D}$. degree granting, in Automatics and Computers Science fields. He also received the special category of Merit Professor from the University of Oriente in 2009 . 